\title{
OBSERVATIONS OF INTERSTELLAR LYMAN- $\alpha$ WITH THE ORBITING ASTRONOMICAL OBSERVATORY
}

\author{
B. D. SAVAGE and A. D. CODE \\ Space Astronomy Laboratory, Washburn Observatory, \\ University of Wisconsin, Madison, Wis., U.S.A.
}

\begin{abstract}
The equivalent width of the blended line at Lyman $\alpha$ is given for 48 stars measured with the OAO-A2 scanning spectrometer. This provides an upper limit to the neutral hydrogen column density. In the Orion association these upper limits are significantly lower than the column densities determined from $21-\mathrm{cm}$ emission line measurements. The determination of the Lyman $\alpha$ equivalent width for $\theta$ Ori by Carruthers is rediscussed and agreement between $21-\mathrm{cm}$ absorption measures and Lyman $\alpha$ absorption is obtained for a spin temperature in the range of $40-70 \mathrm{~K}$. It is suggested that the most likely explanation for the discrepancies found for the other Orion stars is that the $21-\mathrm{cm}$ emission primarily occurs beyond the Belt stars.

The correlation between the OAO blended equivalent widths and color excess, $4430 \AA$ absorption, and interstellar sodium absorption are examined. Excellent correlation between sodium and hydrogen column densities is found.
\end{abstract}

\section{Introduction}

The short wavelength photoelectric scanning spectrometer on board OAO-A2 has scanned approximately 60 early-type stars (as of June 1969) from $1050 \AA$ to $1800 \AA$. The instrumental resolution is $15 \AA$ full width at half maximum. Some 50 of these stars have sufficient flux at $1200 \AA$ to permit measurements of the equivalent width of the interstellar Lyman $\alpha$ line. A single observation consists of two scans and many objects were observed several times. Comparison of scans of the same stars separated by time intervals of several months agree to within $2 \%$. Several representative scans in the region of Lyman $\alpha$ are shown in Figure 1, where the measured digital counts are plotted as a function of wavelength. The counting statistics on a single scan are quite good. The relative weakness of Lyman $\alpha$ in $\varepsilon$ Per and the great strength in $\theta$ Ori is almost entirely due to the interstellar contribution. The variation of the strongest stellar line features with spectral type and luminosity has been described by Code and Bless (1969). A small background count of the order of ten percent of the continuum intensity near Lyman $\alpha$ has been subtracted from the scans in Figure 1. This background is primarily due to scattered light and has been determined from measurements made shortward of $1050 \AA$ where only scattered light and dark counts are recorded. The sky background does not produce a significant contribution on the sensitivity range on which these spectra have been recorded. Any variation in the details of weak spectral features on repeated scans of the same object is due to the discrete nature of the spectrometer steps. The grating is rotated by a stepping motor in $10 \AA$ steps. Since the spectrometer has an objective grating, a variation in absolute pointing of the spacecraft from one set of observations to another of $30 \mathrm{sec}$ of arc may occur corresponding to a displacement of $2.5 \AA$. This results in a modification of the line shapes but has little effect on the equivalent widths. It is unlikely that photometric 
errors, background counts, and spacecraft motions introduce an error in the equivalent widths of strong lines larger than $10 \%$. However, the uncertainty of the continuum level is likely to be the main source of error in the equivalent width measurements.

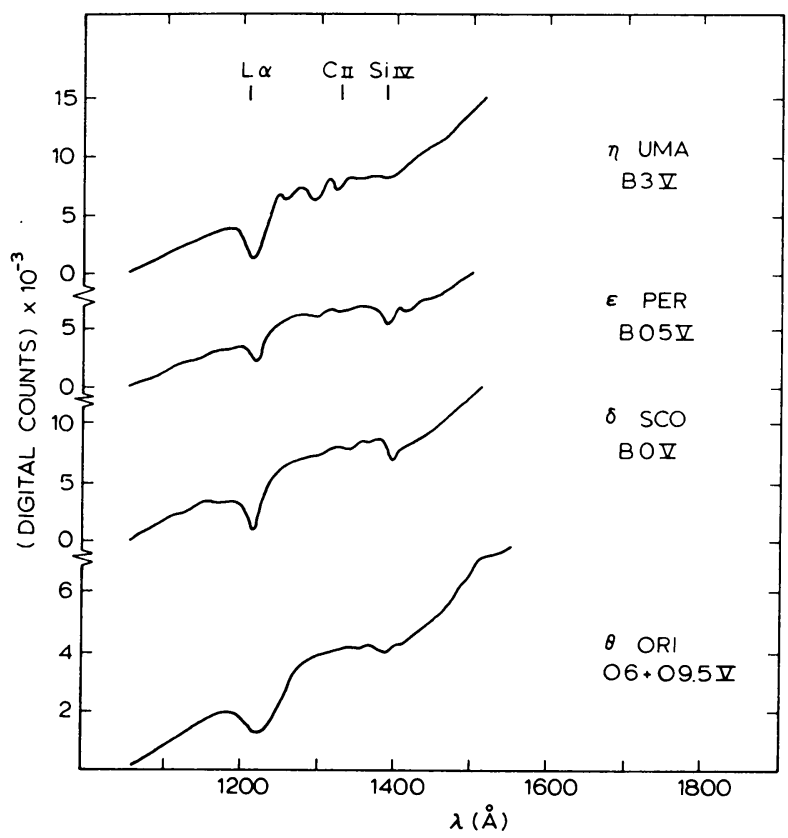

Fig. 1. Spectrometer scans in the region of Lyman $\alpha$ of 4 early-type stars made with the OAO-A2 short wavelength scanner. Digital counts are plotted vs. wavelength.

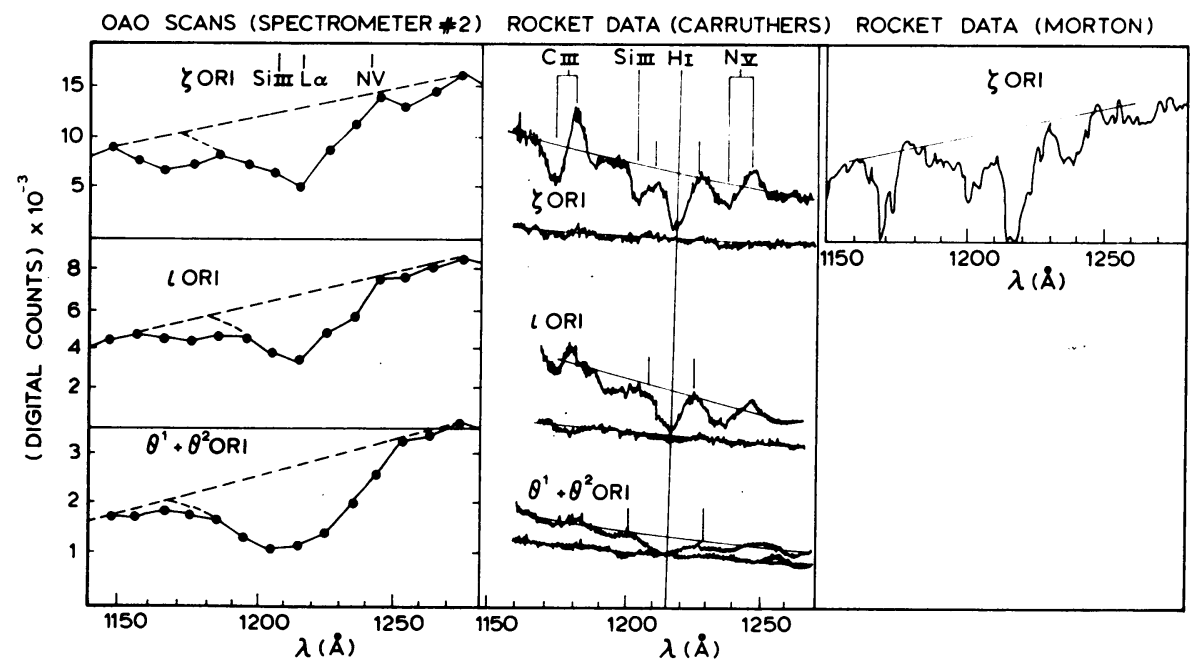

Fig. 2. A comparison of OAO-A2 scanner observations with rocket observations of Carruthers (1969) and Morton (1969). Dashed lines on OAO scans indicate adopted continuum level and portion of Lyman $x$ blend which is measured. For detailed information about the stars see Table I. 
B. D. SAVAGE AND A.D. CODE

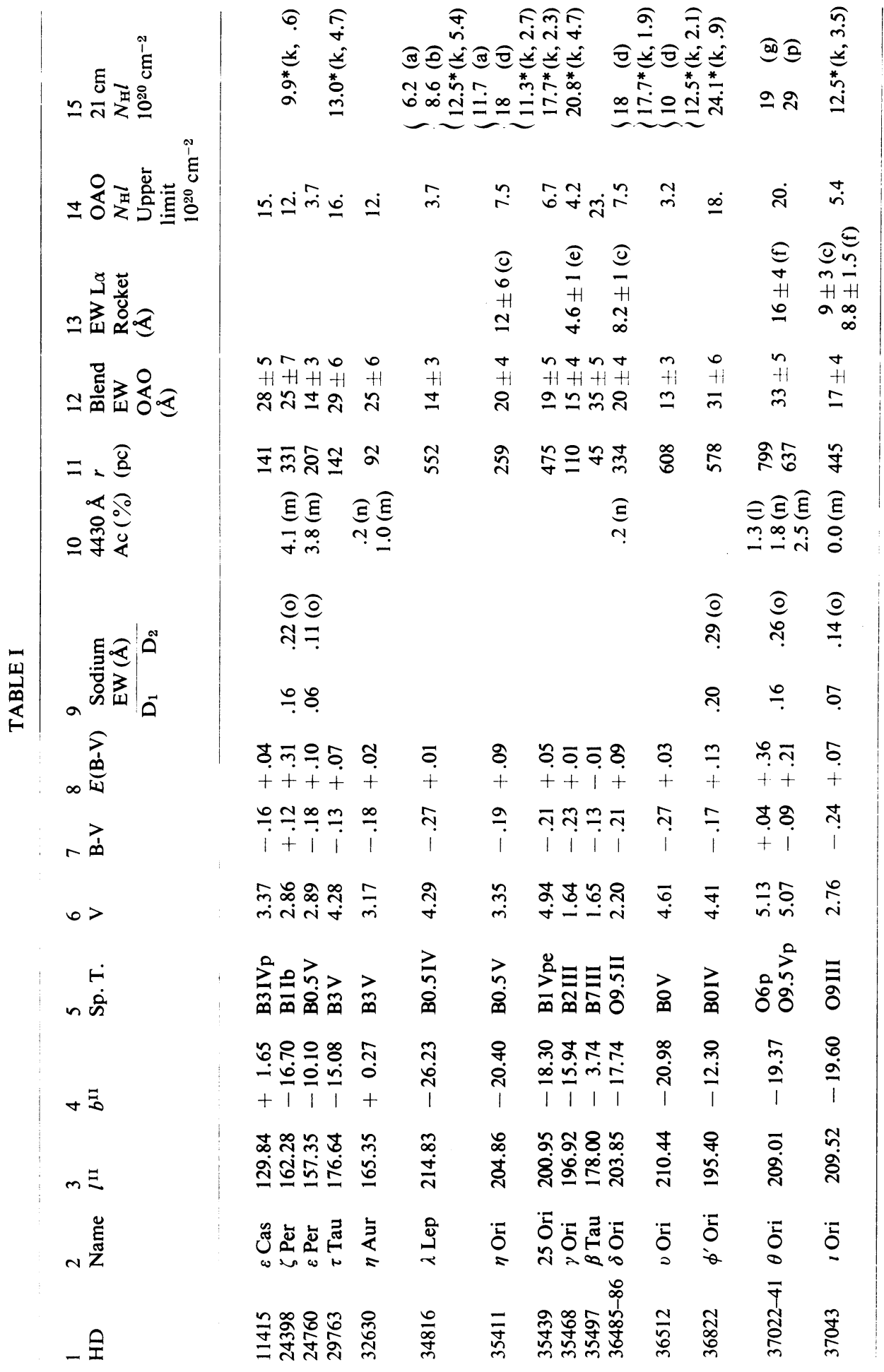




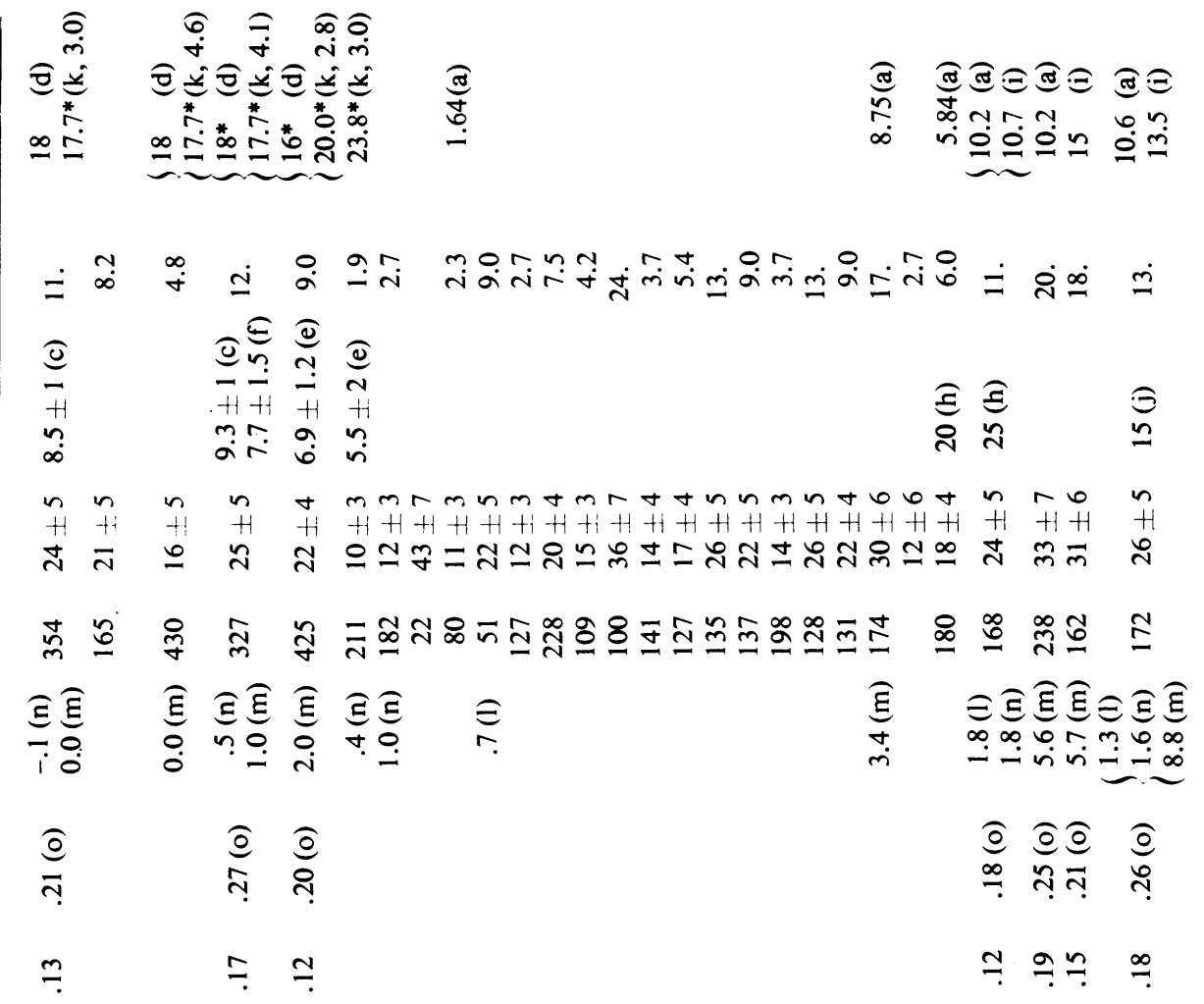

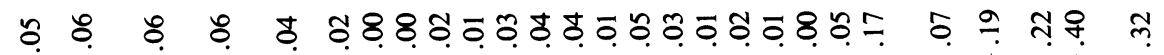

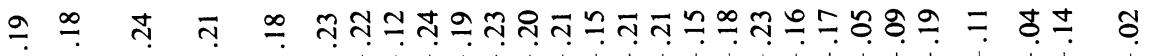

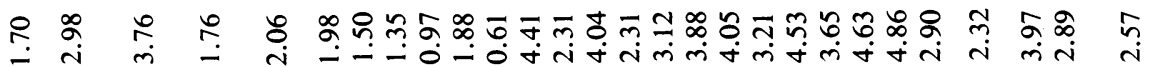

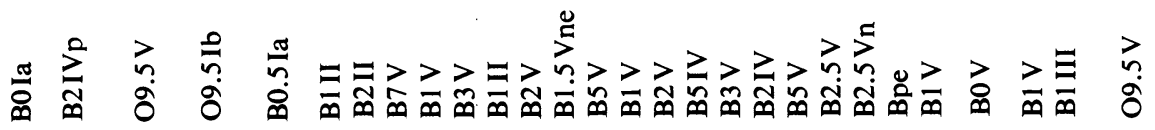

ๆ

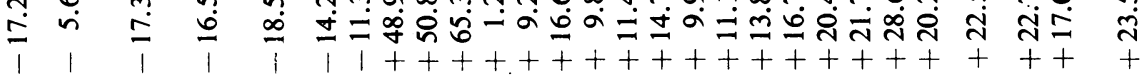

సิ

享

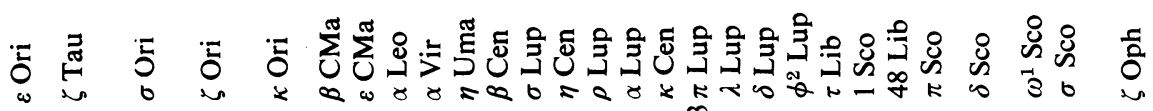

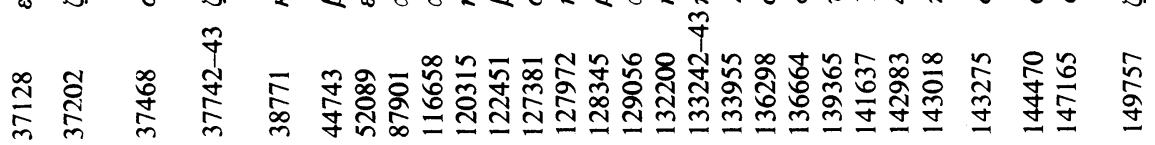




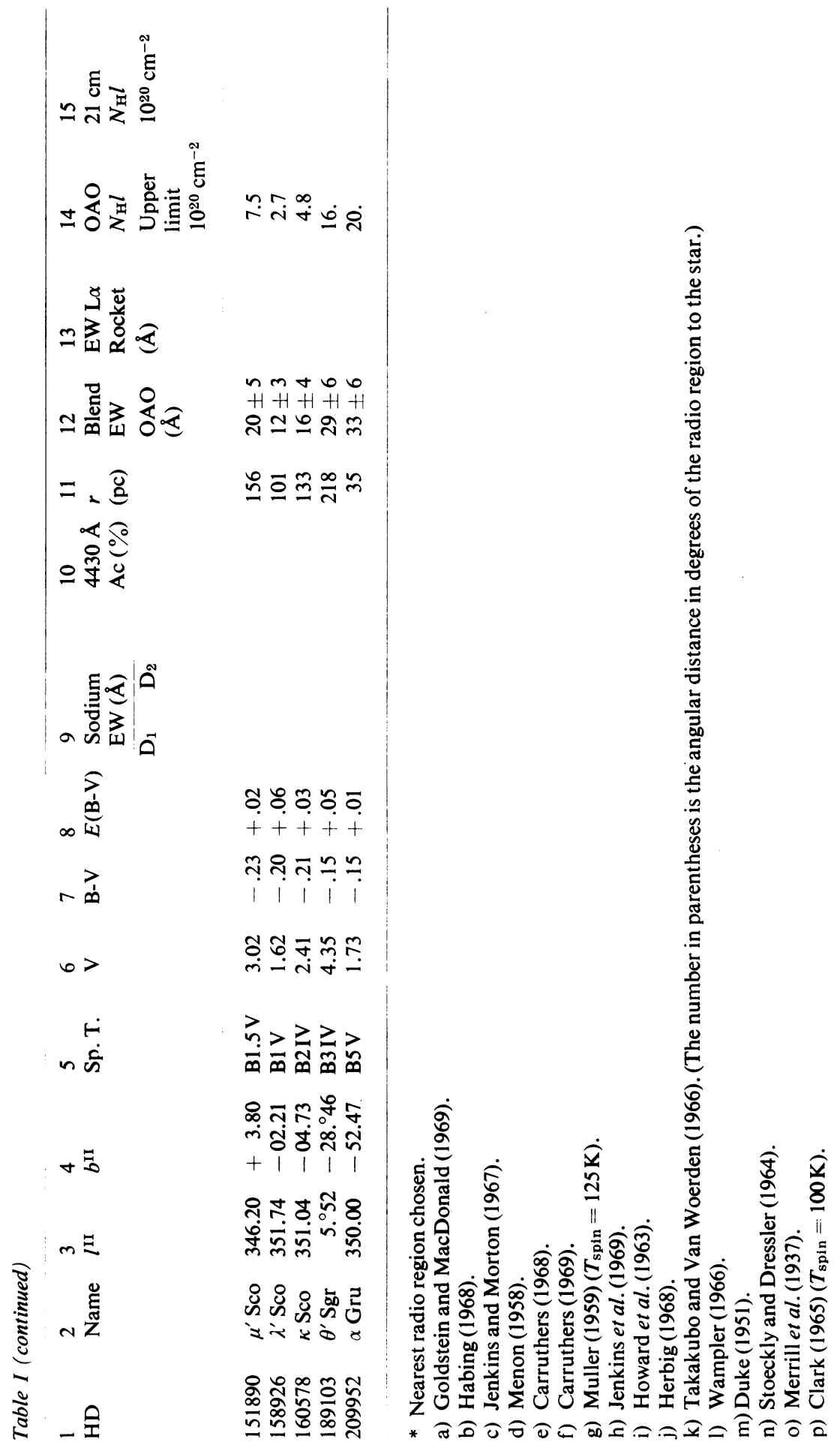


The OAO resolution of $15 \AA$ blends the interstellar Lyman $\alpha$ line with the stellar lines of Si III 1206.5 $\AA$ and Nv 1238.5 $\AA, 1242.8 \AA$ and for stars earlier than O9.5 a stellar feature at approximately $1195 \AA$. A number of higher resolution spectra have been obtained by Jenkins and Morton (1967) and Carruthers (1969) which show these stellar contributions. Figure 2 shows a comparison of the OAO data with rocket data of Carruthers (1969), which is plotted on a density scale and of Morton (1969) on an intensity scale. It should be possible to separate out the effects of the stellar contribution to the blended Lyman $\alpha$ line by means of high resolution scans of brighter stars along with the large number of OAO scans of diverse types and amounts of interstellar absorption. In this preliminary report, however, we merely present measurements of the blend equivalent widths at Lyman $\alpha$. It will be seen that interesting results can be obtained from such measurements. Such measurements provide an upper limit to the column density of neutral hydrogen, which is interesting because the discussions to date (Carruthers 1968, 1969; Jenkins et al., 1967, 1969) have indicated that the hydrogen densities determined from Lyman $\alpha$ measurements were in general considerably smaller than has been inferred from $21-\mathrm{cm}$ data.

Table. I lists the equivalent widths for the OAO line blend at Lyman $\alpha$ for 48 stars, along with other pertinent data. We estimate the possible error to be approximately $\pm 20 \%$, the main uncertainty being due to the continuum level estimate. Column 9 gives the equivalent widths of $\mathrm{Na} \mathrm{D}_{1}$ and $\mathrm{D}_{2}$ lines from Merrill et al. (1937), while column 10 lists the central depths of the diffuse $4430 \AA$ band from several sources. The distances given in column 11 are spectrophotometric distances derived from the spectral types and photometric data given in columns 5, 6, and 7. Column 12 gives the OAO equivalent widths of Lyman $\alpha$ and column 13 the published equivalent widths of Lyman $\alpha$ from rocket data. Column 14 gives the upper limit to the neutral hydrogen column density from the OAO observations. These upper limits were obtained assuming radiation damping and therefore we used the relation $N l=1.865 \times 10^{18} W_{\lambda}^{2}$ atoms $\mathrm{cm}^{-2}$, with $W_{\lambda}$ expressed in $\AA$ (Morton, 1967). The final column gives the $21-\mathrm{cm}$ column densities in the direction of these stars. Except for $\theta$ Ori the $21-\mathrm{cm}$ data refer to emission measurements. In the case of the emission measurements no attempt has been made to separate out emission in front and behind the star (see Jenkins et al., 1969).

\section{Comparison of OAO Data with Rocket Data}

An examination of Table I shows that for all the stars for which rocket data exist, except the Scorpius stars, the OAO blend equivalent width exceeds the rocket Lyman $\alpha$ equivalent widths by a factor of 2 to 3 . In the case of the Scorpius observations of Jenkins et al. (1969), however, the OAO blend equivalent width is approximately equal to their measured Lyman $\alpha$ equivalent width. These latter observations were of resolution comparable to the $\mathrm{OAO}$ resolution, and it appears that their measurements may include some blending with Si III $1206.5 \AA$.

It appears unlikely to us that the SiII and $\mathrm{Nv}$ contributions to the OAO blend could cause a factor of 2 to 3 increase in the OAO blend equivalent width over the 
rocket Lyman $\alpha$ measurements at higher resolutions. Indeed for all of these objects the equivalent widths of the stellar lines account for only about one third of the discrepancy, as judged from the higher resolution spectra. We believe that a more likely explanation of the difference is that in the rocket measurements the continuum level has been systematically underestimated and furthermore inadequate allowance has been made for the broad damping wings of the interstellar Lyman $\alpha$.

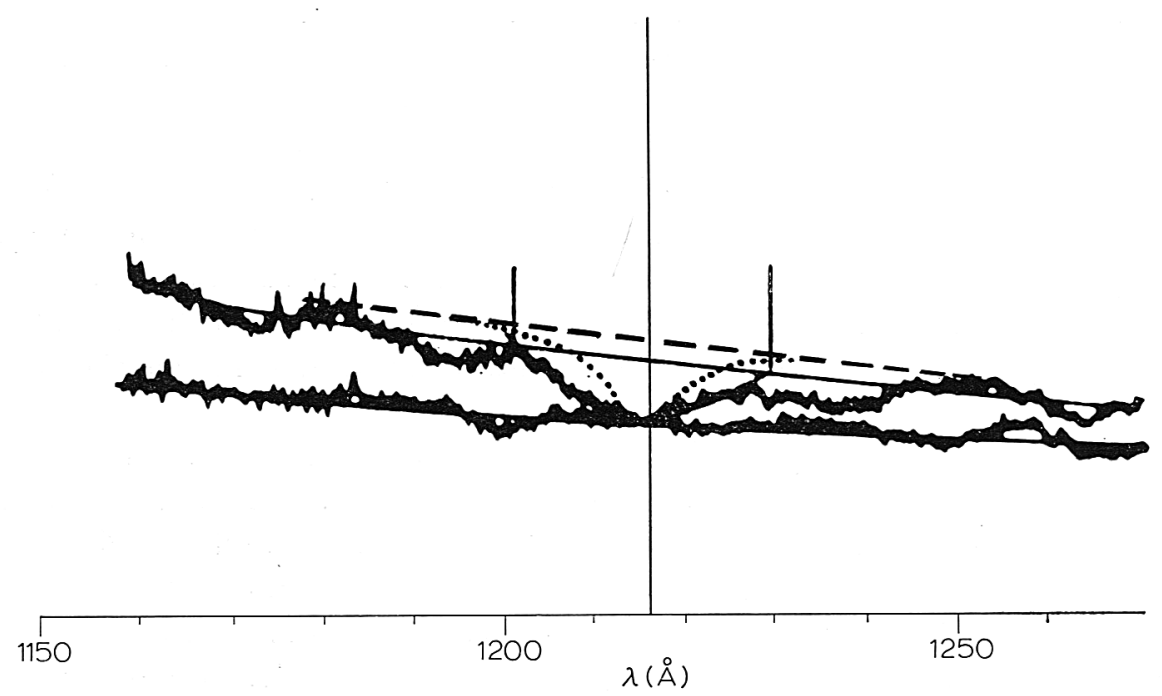

Fig. 3. The $\theta$ Ori observation of Carruthers (1969). Solid line is Carruthers' estimate of the continuum level. We have drawn in a new continuum level (dashed line). The dotted line is the line profile for Lyman $\alpha$ having an equivalent width of $16 \AA$ when referred to the new continuum level.

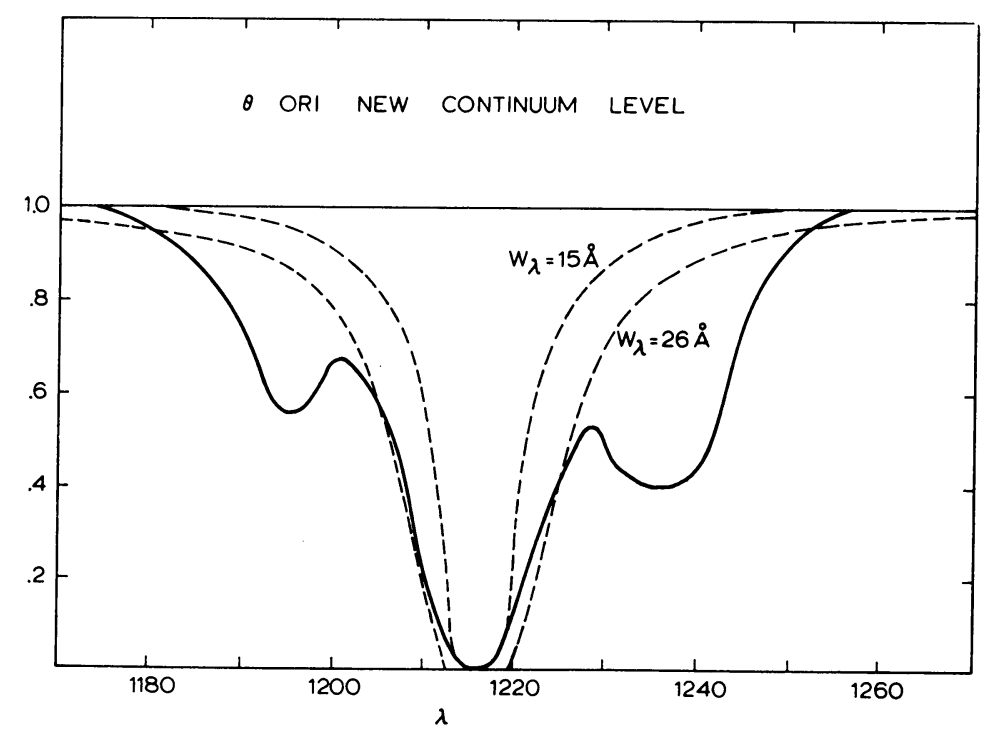

Fig. 4. $\theta$ Ori observation of Carruthers (1969) redrawn using the new continuum level of Figure 3. Lyman $\alpha$ lines with equivalent widths of $15 \AA$ and $26 \AA$ are drawn in for comparison. 
The case of $\theta$ Ori is particularly important since $21-\mathrm{cm}$ absorption measurements exist for the Orion nebula. Carruthers (1969) measured an equivalent width of 16 $\pm 4 \AA$ from his $\theta$ Ori observations. This yields a hydrogen column density of about $\frac{1}{5}$ the value obtained from $21-\mathrm{cm}$ absorption measures assuming a spin temperature of $100 \mathrm{~K}$. He suggests that the discrepancy could be removed if the spin temperature was about $20 \mathrm{~K}$. The OAO blend equivalent width is $33 \pm 5 \AA$. Figure 3 shows the observations of Carruthers (1969) on which a new continuum has been indicated by the dashed line. The three solid lines (two vertical and one horizontal) define the area Carruthers measured in his equivalent width determination of $16 \pm 4 \AA$. We assume in this discussion that, for the low photographic densities recorded in Carruthers' $\theta$ Ori observation, intensity is directly proportional to photographic density (unfortunately there was no discussion of intensity calibration in Carruthers' paper). We have also indicated in Figure 3 for the new continuum level a Lyman $\alpha$ damping profile with an equivalent width of $16 \AA$. As would be expected, for the new continuum level this line poorly matches the observations. However, even when referred to Carruthers' original continuum a $16 \AA$ line profile does not give a good representation of the line shape. In Figure 4 the observations of Carruthers are redrawn using the new continuum of Figure 3 and the Lyman $\alpha$ profiles for an equivalent width of $15 \AA$ and $26 \AA$ are drawn in for comparison. Our estimate of the equivalent width of Lyman $\alpha$ for $\theta$ Ori based on the data in Figure 3 is $24 \pm 8 \AA$. It is of interest that with the new continuum level the blend equivalent width for $\theta$ Ori (as determined from Figure 4 ) is $34 \AA$, in good agreement with the OAO blend measurement. Since the interstellar line

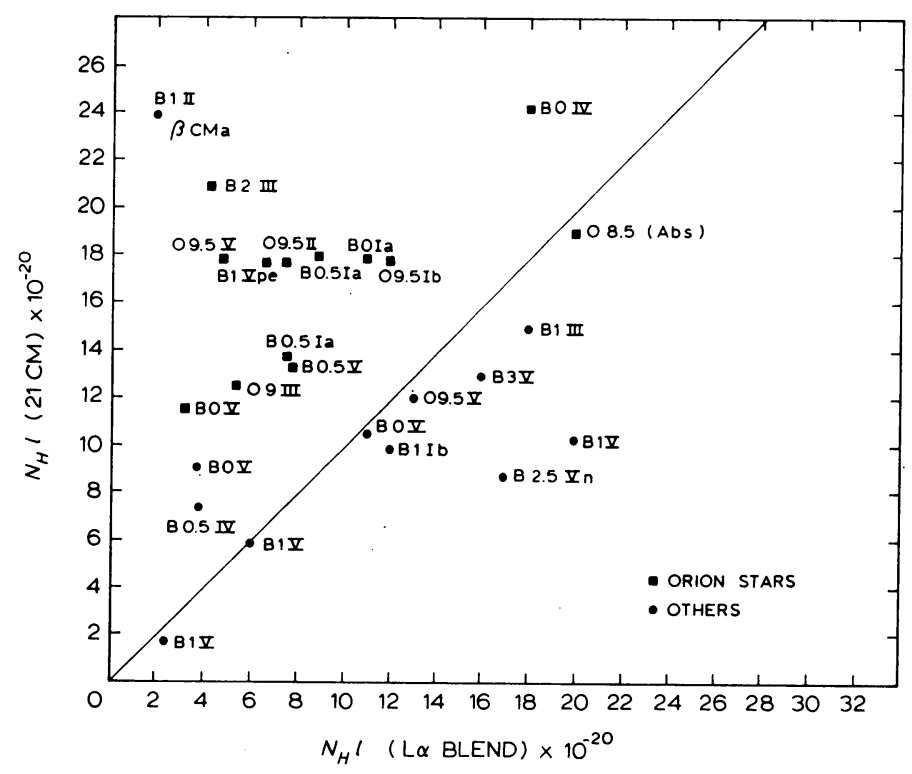

Fig. 5. Correlation between column density obtained from OAO blend equivalent widths (an upper limit to the column density) and the $21-\mathrm{cm}$ radio emission and absorption column densities. Orion stars are indicated with a $\square$, other stars are indicated with a 0 . 
is on the square-root portion of the curve of growth, the $50 \%$ increase in the equivalent width of Lyman $\alpha$ for $\theta$ Ori results in an increase in the column density of more than a factor of 2. The column density for an equivalent width of $24 \AA$ is $N_{\mathrm{H}} l=11 \times 10^{20}$ atoms per $\mathrm{cm}^{2}$. Muller (1958) gives a value of $N_{\mathrm{H}} l=19 \times 10^{20}$ per $\mathrm{cm}^{2}$ from the $21-\mathrm{cm}$ absorption line based on a spin temperature of $125 \mathrm{~K}$. If we equate the Lyman $\alpha$ and $21-\mathrm{cm}$ densities we derive a spin temperature of approximately $70 \mathrm{~K}$. Carrying out the same calculation but using the radio absorption data of Clark (1965) $\left(N_{\mathrm{H}} l=2.9 \times 10^{21}\right.$ for $T_{\text {spin }}=100 \mathrm{~K}$ ) we obtain a spin temperature of approximately $40 \mathrm{~K}$.

In Figure 5 the column density determined from $21-\mathrm{cm}$ measures is plotted against the upper limit to the column density given by the OAO blend. Thus correcting the OAO Lyman $\alpha$ measurements for the effects of blending would move points to the left. The Orion stars are plotted as squares, the other stars as circles. Excluding the Orion stars, the $21-\mathrm{cm}$ densities are usually somewhat less than the OAO upper limit

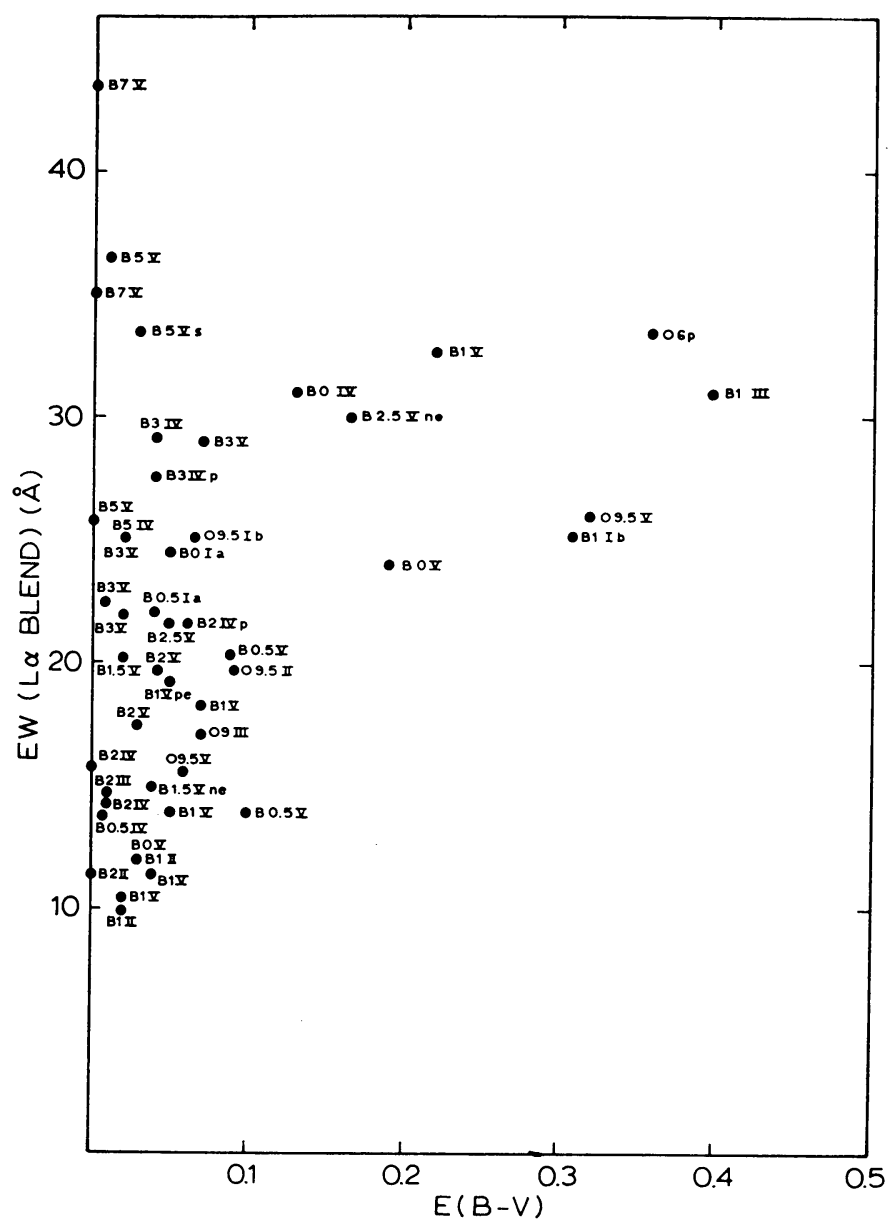

Fig. 6. Correlation between Lyman $\alpha$ blend equivalent width and color excess. The spectral type of each star is indicated on the figure. 
as would be expected if most of the $21-\mathrm{cm}$ emission is not background emission. In Orion the $21-\mathrm{cm}$ column densities range from 1.5 to 5 times the OAO upper limit. In view of the reasonable agreement found for the $21-\mathrm{cm}$ absorption measurement in front of the Orion Nebula and the Lyman $\alpha$ absorption for $\theta$ Ori it is reasonable to believe that most of the $21-\mathrm{cm}$ emission in the direction of the Orion association occurs at distances greater than the bright Orion stars.

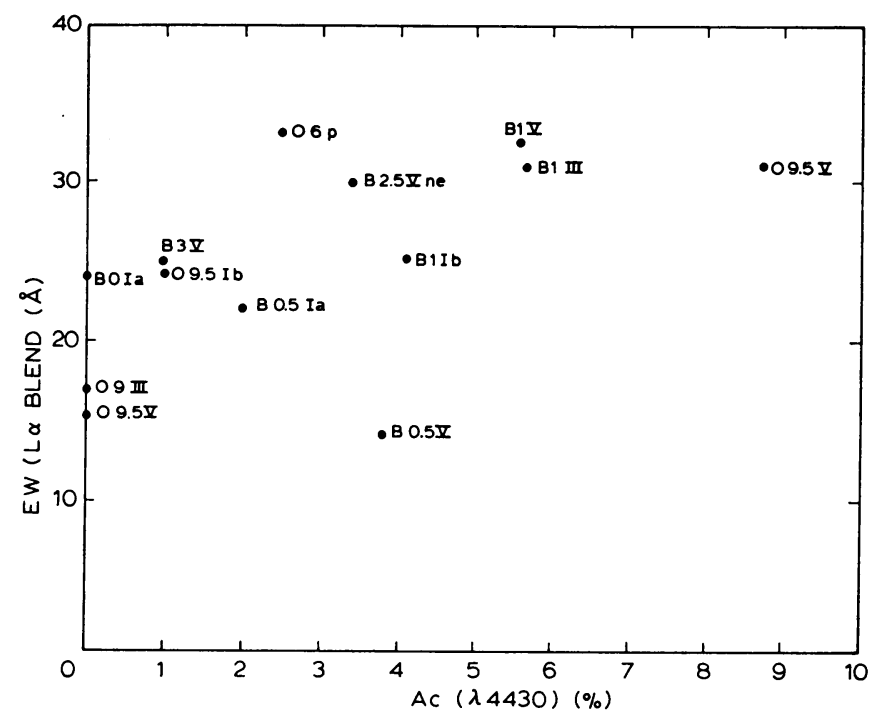

Fig. 7. Correlation of the equivalent width of the Lyman $\alpha$ blend with the $4430 \AA$ central absorption measurements of Duke (1951).

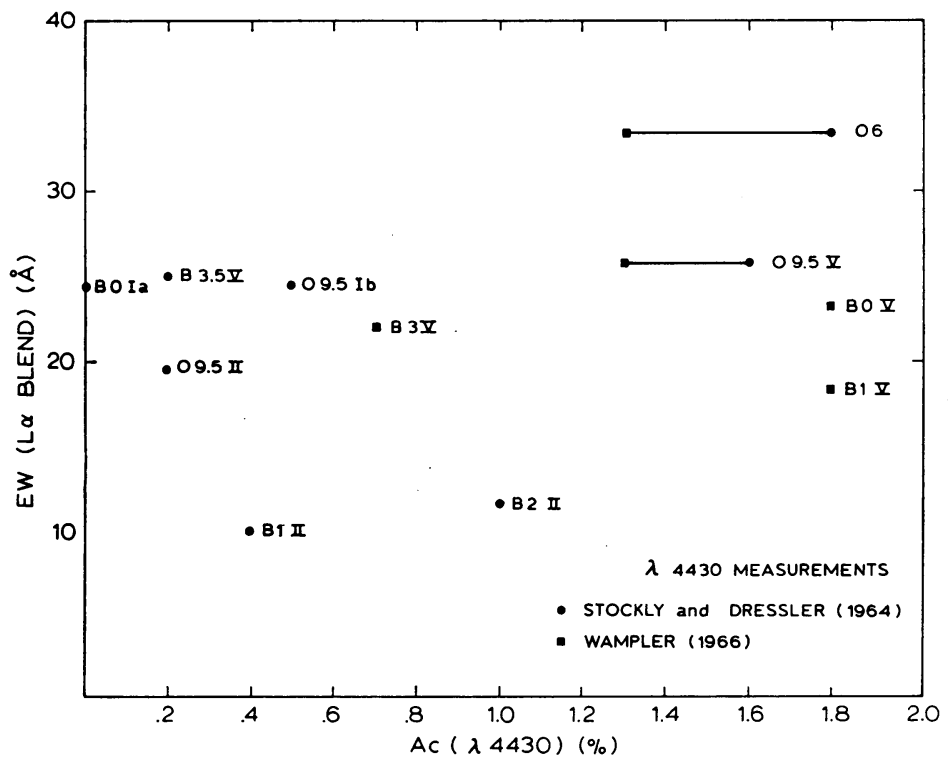

Fig. 8. Correlation of the equivalent width of the Lyman $\alpha$ blend with the $4430 \AA$ central absorption measurements of Stoeckly and Dressler (1964), and Wampler (1966). 


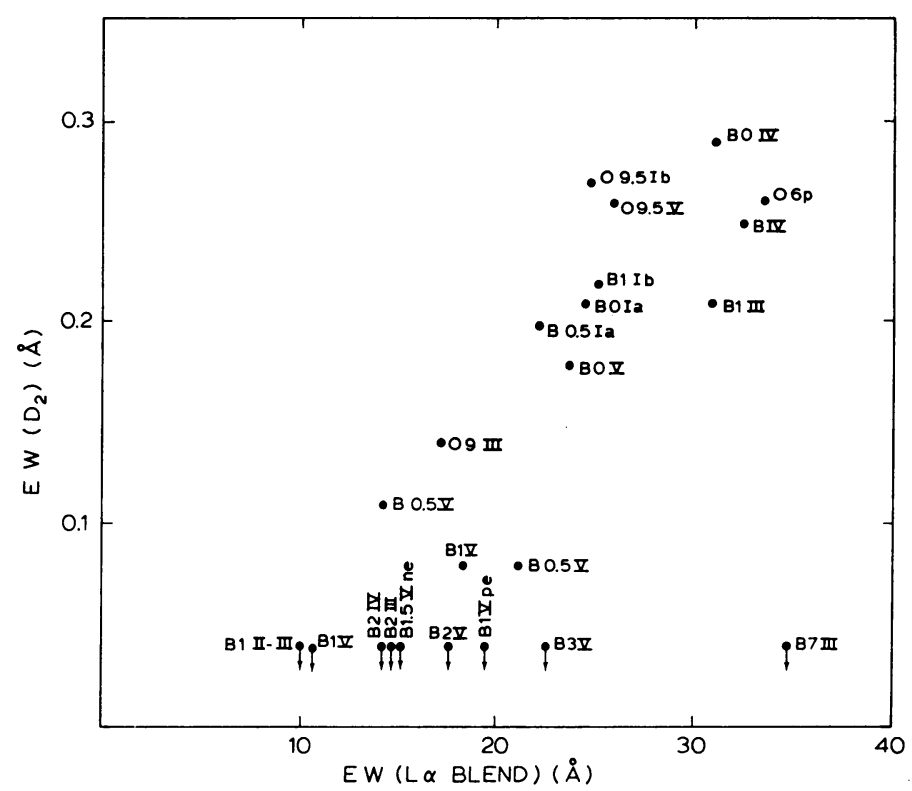

Fig. 9. Correlation of the equivalent width of the Lyman $\alpha$ blend with the equivalent width of the sodium $\mathrm{D}_{2}$-line. Points connected to arrows indicate the upper limit on $\mathrm{D}_{2}$ equivalent width. Sodium data is from Merrill et al. (1937).

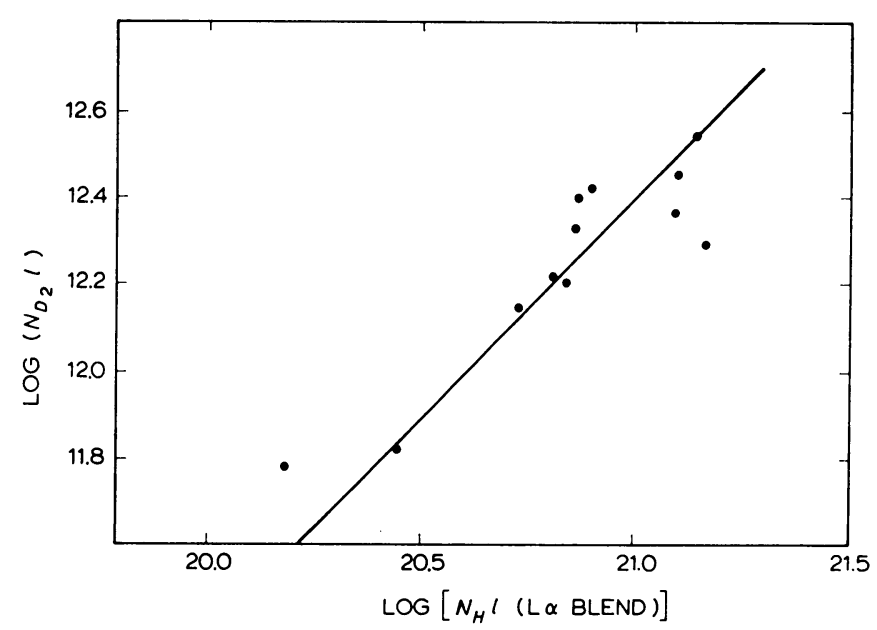

Fig. 10. Column density of atomic hydrogen (obtained from the Lyman $\alpha$ blend) vs. column density of neutral sodium atoms.

\section{Correlations of Blend with Other Interstellar Features}

In Figure 6 the equivalent width of the OAO Lyman $\alpha$ blend is plotted against the $B-V$ color excess of the stars. The early-type stars do show a general correlation of increasing equivalent width with increasing color excess. For stars with small color 
excesses the equivalent width shows a systematic increase with spectral type. This is a result of the increasing strength of the stellar lines included in the blend. In particular Si III and stellar Lyman $\alpha$ become quite strong in stars later than B3. For B0 V stars the stellar lines contribute 5 to $10 \AA$ to the measured equivalent widths. A simple subtraction, however, cannot be used to go from the Lyman $\alpha$ blend equivalent width to the interstellar Lyman $\alpha$ equivalent width.

In Figures 7 and 8 the equivalent width of the Lyman $\alpha$ blend is plotted against measurements of the central absorption of the $4430 \AA$ diffuse interstellar band. Figure 7 shows the data of Duke (1951) and Figure 8 the measurements by Stoeckly and Dressler (1964), and Wampler (1966). The correlation is poor, just as that between the $4430 \AA$ band and the sodium D-line is known to be.

Figure 9 shows the correlation of the blend equivalent width with the equivalent width of the sodium $\mathrm{D}_{2}$ line from the data of Merrill et al. (1937). The correlation between these data is very good. The stars plotted are located in Orion, Scorpius, and Perseus. The stars at the bottom of the illustration connected to arrows are stars for which interstellar D is very weak or absent. Among these stars we see the increasing equivalent width of the blend with spectral type indicating the growth of the stellar contribution. Sodium column densities were determined for these stars from the doublet ratio and the results are plotted in Figure 10. The agreement between the $\mathrm{D}_{2}$ column densities and the hydrogen densities is good and in reasonable agreement with abundance ratios and ionization estimates.

\section{Conclusion}

The discussion presented above is preliminary in nature but suggests the direction which future work might take. Additional OAO Lyman $\alpha$ measurements are being made. We are attempting to extract the effect of the blended stellar lines both on the basis of higher resolution profiles and on the differential comparison of stars of the same spectral type. Clearly it would be helpful to have additional data on interstellar absorption for these stars, both radio data and optical measurements of interstellar $\mathrm{Ca}, \mathrm{Na}, \mathrm{CH}, 4430 \AA$, etc., would be useful.

\section{References}

Carruthers, G.: 1968, Astrophys. J. 151, 269.

Carruthers, G.: 1969, Astrophys. J. 156, L97.

Clark, B. G.: 1965, Astrophys. J. 142, 1398.

Code, A. D. and Bless, R. C.: 1969, this volume, p. 173-177.

Duke, D.: 1951, Astrophys. J. 113, 100.

Goldstein, S. J. and MacDonald, D.: 1969, Astrophys. J., in press.

Habing, H. J.: 1968, Bull. Astron. Inst. Netherl.20, 120.

Herbig, G. H.: 1968, Z. Astrophys. 68, 243.

Howard, W. E., Wentzel, D. G., and McGee, R. X.: 1963, Astrophys. J. 138, 988.

Jenkins, E. B. and Morton, D. C.: 1967, Nature 215, 1257.

Jenkins, E. B., Morton, D. C., and Matilsky, T. A.: 1969, in press.

Menon, T. K.: 1959, Astrophys. J. 127, 28. 
Merrill, P. W., Sanford, R. F., Wilson, O. C., and Burwell, C. G.: 1937, Astrophys. J. 86, 274. Morton, D. C.: 1967, Astrophys. J. 147, 1017.

Muller, C. A.: 1959, Proc. IAU Symp. 9, Stanford Univ. Press, Stanford, Calif., p. 360.

Stoeckly, R. and Dressler, K.: 1964, Astrophys. J. 139, 240.

Takakubo, K. and Van Woerden, H.: 1966, Bull. Astron. Inst. Netherl. 18, 488.

Wampler, E. J.: 1966, Astrophys. J. 144, 921.

\section{Discussion}

Carruthers: You mentioned that the equivalent width of your 'blend' is about a factor of two greater than our equivalent width of the Lyman $\alpha$ line for $\theta$ Ori. I would like to point out that the $\mathrm{Nv}$ line is very strong in an $\mathrm{O6}$ star, in the case of $\theta$ Ori this line is comparable to or possibly even stronger than the Lyman $\alpha$ line, hence the combined strength of both lines would be in good agreement with your measurements.

Bless: $\mathrm{Nv}$ is indeed strong in $\theta$ Ori but the factor of 2-3 refers to all the OAO-rocket comparisons, except those in Scorpius recently observed by Princeton. This includes stars in which $\mathrm{Nv}$ is not very strong.

Carruthers: Would you agree that it is possible to raise the level of your continuum as I suggested earlier?

Bless: Yes, I agree that there is room for raising the continuum. Our spectrum of $\theta$ Ori is rather weak in this wavelength range and there is an appreciable uncertainty in the continuum level.

Greenberg: If we believe in a generally good correlation of $4430 \AA$ with extinction, how do you reconcile your observation of lack of correlation of Lyman $\alpha$ and $4430 \AA$ depth with the result which Jenkins presented showing an almost surprisingly excellent correlation of Lyman $\alpha$ with extinction?

Bless: I don't know. The correlation - if it exists - might be masked by our measuring a blend, rather than Lyman $\alpha$ alone. Also, we have observed relatively few stars for which $4430 \AA$ measurements exist.

Morton: Is it possible to reduce the problems of the blends by concentrating on stars of types B1 to B3 where the contributions of $\mathrm{Nv}$ and Sill may be rather small without too serious a contribution from the photospheric Lyman $\alpha$ line?

Bless: Yes, this is one of the things we intend to do to sort out the effects of the blend.

Morton: Since the OAO Lyman $\alpha$ data tend to agree with the rocket observations toward Orion, would the radio astronomers object to a real hole in the HI density in this direction?

Oort: It is perhaps not implausible to think that the interstellar gas in the Orion region would form some sort of a relatively thin sheet. If this is supposed to be related to the Orion association one could easily imagine that there would be holes in the neutral hydrogen around the high-temperature stars while the rest of the sheet would remain neutral. In such case the Lyman $\alpha$ absorption results for the $\mathrm{HI}$ density might well be much lower than those derived from the $21-\mathrm{cm}$ emission measurements.

Bless: Since OAO observations suggest that for some stars in other parts of the Galaxy there is reasonable agreement between Lyman $\alpha$ and $21 \mathrm{~cm}$ measurements, such a local density anomaly in the Orion region seems a little more plausible than looking for fundamental difficulties with $21-\mathrm{cm}$ observations, etc. 